\title{
Ein geheimes Netzwerk zur Vorbereitung und Durchführung des Attentats- und Staatsstreichversuchs vom 20. Juli 1944
}

\author{
von \\ LINDA VON KEYSERLINGK
}

\begin{abstract}
Das Netzwerk vom 20. Juli 1944 war ein gebeimes Kommunikationsnetzwerk zwischen zivilen und militärischen Regimekritikern mit dem Ziel, das NS-Regime zu stürzen und einen neuen Rechtsstaat aufzubauen. Während sich einige Teilgruppen bereits ab 1933 in geheimen Strukturen zu organisieren begannen, suchten andere erst Ende der 1930er Jahre oder während der Kriegsjabre nach Verbündeten. Alle Beteiligten waren durch ibr Wissen und ibr Mitwirken an der Attentats- und Staatsstreichvorbereitung in existentieller Weise durch die Gestapo und in letzter Konsequenz durch die NS-Justiz bedroht. Die Geheimhaltung wurde somit zur Voraussetzung für das Vorhaben und prägte die Kommunikationsformen ebenso wie die Struktur und die Funktionalität des Netzwerkes. Die notwendigen Vorsichtsmaßnahmen wirkten sich außerordentlich hemmend für die effektive Vorbereitung des Umsturzes aus. Viele Beteiligte waren aus Sicherheitsgründen nicht miteinander bekannt. Dennoch gab es unzählige Verbindungen innerhalb und zwischen den einzelnen Gruppen, die häufig eine ausgesprochen hohe Intensität aufwiesen. Das Netzwerk des 20. Juli 1944 war durch eine gewisse Zentralisation geprägt, verfügte jedoch über keine strenge hierarchische Struktur. Die militärinterne Hierarchie - verstärkt durch den Eid zu bedingungslosem Gehorsam - stellte die Verschwörer hingegen vor größere strukturelle Schwierigkeiten.
\end{abstract}

Das Netzwerk der Verschwörer des 20. Juli 1944 war ein konspiratives Kommunikationsnetz, welches ab 1938/39 zu dem Zweck der Vorbereitung eines Attentats- und Staatsstreichversuches sowie eines darauf folgenden neuen Staatsaufbaus entstand. Bis 1944 wurde es stetig weiter ausgebaut. Die für alle Beteiligten überlebenswichtige Geheimhaltung des Vorhabens führte jedoch zu einem nicht unwesentlichen Dilemma: Während es für das Gelingen des Staatsstreiches und des darauf folgenden Aufbaus einer neuen Regierung möglichst vieler Mitverschwörer, einer zuverlässigen Struktur und einer breiten Basis der Unterstützung bedurfte, musste die Zahl der Eingeweihten und der Grad ihrer Involvierung aus Sicherheitsgründen so weit wie möglich begrenzt bleiben. Diese beiden konträren Wirkungseinflüsse erschwerten die Kommunikation und die oppositionelle Arbeit erheblich. Jede Erweiterung des Netzwerkes um neue Mitverschwörer und Mitverschwörerinnen erhöhte zwar die Aussicht auf Erfolg, zugleich aber auch die Gefahr, verraten und vom NSRegime erkannt und verfolgt zu werden. Die Überlegung, wer zu welchem Zeitpunkt in welchem Umfang eingeweiht werden sollte, war somit sowohl 
für das Gelingen des Vorhabens als auch für den Schutz aller Beteiligten von existenzieller Bedeutung.

Um das NS-Regime stürzen zu können, bedurfte es nicht nur eines Attentäters, der den Diktator ausschaltete, sondern auch zahlreicher eingeweihter Personen, die im Hintergrund wirkten. Der Ablauf des Staatsstreiches musste konkret geplant und Sprengstoff beschafft werden. Zuverlässige Truppenteile mussten bereitgestellt werden, um die NS-Führung zu verhaften, um wichtige Schaltzentralen zu besetzen und um die Verschwörer vor Gegenangriffen zu schützen. Die Westalliierten sollten informiert und für die neue Regierung sowie für ein Friedensangebot gewonnen werden ${ }^{1}$. Um einen drohenden Bürgerkrieg zu verhindern, bedurfte es einer guten Vorbereitung des geplanten neuen Rechtsstaates. Weder ein Einzelner, noch eine einzelne soziale Gruppe war dabei in der Lage, diese Aufgaben selbständig zu bewältigen. Ohne die Militärs konnten Attentat und Umsturz nicht gelingen. Doch ohne die diplomatischen Beziehungen ins Ausland, ohne die Sachkenntnis ziviler Experten aus allen Gesellschaftsbereichen und ohne eine breite Basis in der Bevölkerung konnten keine neue Regierung und kein neuer Rechtsstaat aufgebaut werden.

Diese für einen Umsturz erforderliche und auch realisierte soziale, berufliche und politische Vielfalt der Beteiligten, wurde von der NS-Führung zunächst nicht erkannt. Die frühen Gestapo-Auflistungen, die im Zuge der Ermittlungen nach dem Attentat am 20. Juli 1944 entstanden, nannten ausschließlich Berufs- und Reserveoffiziere als Beteiligte des Attentats- und Umsturzversuches $^{2}$. Doch schon bald stellten die Gestapo-Beamten fest, dass es sich keineswegs um eine rein militärische Verschwörung gehandelt hätte, sondern um eine Bewegung, in der auch zivile Oppositionelle eng mit eingebunden gewesen waren. In einem Schreiben des Chefs des Sicherheitsdienstes (SD), SS-Obergruppenführer Ernst Kaltenbrunner, an Hitlers Sekretär Martin Bormann vom 9. August 1944 ist zu lesen: Bei den Untersuchungen stellt sich immer klarer heraus, dass sich die Verschwörerclique deutlich in zwei Kreise geschieden hat, der militärische Kreis um Stauffenberg und der zivile Kreis um Goerdeler. Allerdings bestand zwischen den beiden Personenkreisen keinerlei scharfe Trennung, sondern die Beziehungen liefen vielfach ineinander. Alle wesentlichen Personen kannten sich, und beispielsweise haben sich sowohl Goerdeler als auch Stauffenberg gewisser Verbindungen gemeinsam bedient ${ }^{3}$." In den Verhören und durch die Ermittlungen stellten sie immer mehr Beziehungen zu Zivilisten in verschiedensten Positionen fest. Schon bald zeigte sich, dass neben

Hierzu vgl. u. a. Klemens von Klemperer, Die verlassenen Verschwörer. Der deutsche Widerstand auf der Suche nach Verbündeten 1938-1945, Berlin 1994.

2 »Spiegelbild einer Verschwörung «. Die Opposition gegen Hitler und der Staatsstreich vom 20. Juli 1944 in der SD-Berichterstattung. Geheime Dokumente des ehemaligen Reichssicherheitshauptamtes, hrsg. von Hans-Adolf Jacobsen, Stuttgart 1984, Bericht vom 24.7.1944, S. 16. Genannt wurden: Generaloberst a.D. Ludwig Beck, General der Infanterie Friedrich Olbricht, die Brüder Marineoberstabsrichter Berthold und Oberst Claus Schenk Graf von Stauffenberg, Oberst Albrecht Ritter Mertz von Quirnheim, Oberleutnant der Reserve Fritz-Dietlof von der Schulenburg, Oberleutnant der Reserve Werner von Haeften, Generalfeldmarschall Erwin von Witzleben und Generaloberst Erich Hoepner; vgl. auch ebd., S. 18, 23.

Ebd., S. 177. 
den Militärs auch Ministerialbeamte, Kirchenvertreter, Sozialisten, Gewerkschafter, Einzelpersonen des öffentlichen Lebens oder auch Mitglieder unterschiedlicher gesellschaftlicher Kreise wichtige Vertreter der Umsturzbewegung gewesen waren ${ }^{4}$.

Der 20. Juli 1944 ist daher als rein militärischer Widerstand nicht denkbar, da die beteiligten Militärs nicht ohne die zivilen Kreise agieren konnten und wollten. Nicht zuletzt deshalb werden in der Forschung mitunter auch Begriffe wie »Militäropposition « und » militärischer Widerstand « im Zusammenhang mit dem 20. Juli 1944 abgelehnt ${ }^{5}$. Daher können in diesem Artikel die zivilen Mitverschwörer des Umsturzversuches nicht unbeachtet bleiben, auch wenn sich der vorliegende Themenband explizit geheimen militärischen Netzwerken widmet.

Die Forschung zum 20. Juli 1944 umfasst eine Vielzahl von Biographien, Darstellungen einzelner Widerstandskreise, Untersuchungen zum Hergang der Ereignisse sowie zu den Motiven und Zielen der Beteiligten ${ }^{6}$. Doch gibt es bislang keine genauere Analyse über den Aufbau des Netzwerkes der Beteiligten ${ }^{7}$. Eine klare Abgrenzung des Netzwerkes des 20. Juli 1944 ist nicht ohne weiteres möglich. Die Zahl der zu berücksichtigenden Personen variiert erheblich je nach Wahl des Ausschlusskriteriums. Im Zentrum standen die aktiven Verschwörer, die entweder bereit waren, selbst ein Attentat auszuführen, oder an der Staatsstreichplanung bzw. Konzeption eines neuen Rechtsstaates sowie an der Truppenbereitstellung oder Sprengstoffbeschaffung beteiligt waren. Hinzu kamen jene, die stetig darum bemüht waren, neue Mitverschwörer zu gewinnen und zwischen den einzelnen Gruppen zu vermitteln. Andere haben dagegen weniger aktiv gewirkt, aber ihre grundsätzliche Bereitschaft gezeigt, nach einem Umsturz ein Amt oder eine bestimmte Funktion zu übernehmen. Des Weiteren kann eine große Gruppe von Mitwissern

Ebd., S. XVII.

Vgl. Klaus-Jürgen Müller, Zur Struktur und Eigenart der nationalkonservativen Opposition bis 1938 - Innenpolitischer Machtkampf, Kriegsverhinderungspolitik und Eventual-Staatsstreichplanung. In: Der Widerstand gegen den Nationalsozialismus. Die deutsche Gesellschaft und der Widerstand gegen Hitler, hrsg. von Jürgen Schmädeke und Peter Steinbach, München u. a. 1994, S. 329; vgl. auch Peter Steinbach, Der militärische Widerstand und seine Beziehungen zu den zivilen Gruppierungen des Widerstandes. In: Aufstand des Gewissens. Militärischer Widerstand gegen Hitler und das NS-Regime 1933-1945, hrsg. von Thomas Vogel, Bonn 2000, S. 49-87, hier S. 51, 60, $73 \mathrm{f}$. Steinbach verweist hier auch auf die Ergebnisse der Arbeiten von Hans Rothfels und Ger van Roon.

6 Vgl. hierzu u. a. die Bibliographie der Gedenkstätte Deutscher Widerstand: <http://www.gdwberlin.de/de/angebote/publikationen/ueberblick > (5. August 2015). Zur Vielfalt des Widerstandes und zu verschiedensten Einzelaspekten vgl. u. a. den umfangreichen Sammelband: Widerstand, hrsg. von Schmädeke/Steinbach (wie Anm. 5). Zum Hergang der Ereignisse vgl. u. a. Peter Hoffmann, Widerstand. Staatsstreich. Attentat. Der Kampf der Opposition gegen Hitler, 4. Aufl., München 1985.

7 Erste Ansätze diesbezüglich sind zu finden in: Klaus-Jürgen Müller, Struktur und Entwicklung der national-konservativen Opposition. In: Aufstand, hrsg. von Vogel (wie Anm. 5), S. 89-133. Vgl. auch Romedio Graf von Thun-Hohenstein, Die Einsamkeit der Mutigen - Von den Schwierigkeiten der Netzwerkbildung im Widerstand. In: Der 20. Juli 1944 - Profile. Motive. Desiderate. XX. Königswinterer Tagung 23.-25. Februar 2007, hrsg. von Stephen Schröder und Christoph Studt, Berlin u. a. 2008, S. 177-191. 
und Sympathisanten dazu gezählt werden. Als Opfer des 20. Juli 1944 müssen auch Personen genannt werden, die zwar am Umsturzversuch unbeteiligt waren, aber aus verschiedenen Gründen, wie beispielsweise Fluchthilfe, ebenfalls verfolgt und teilweise auch hingerichtet wurden. Wird als Auswahlkriterium die aktive Teilnahme am Attentats- und Staatsstreich gewählt, so umfasst die zu berücksichtigende Personengruppe etwa 200 Personen $^{8}$.

\section{Exklusion und Inklusion}

Trotz zahlreicher Differenzen zwischen den verschiedenen Gruppierungen des 20. Juli 1944 einte die Verschwörer das gemeinsame Ziel, das Regime zu stürzen und einen neuen Rechtsstaat aufzubauen'. In der Regierungserklärung, die nach dem Umsturz abgegeben werden sollte, hieß es unter Punkt 1: »Erste Aufgabe ist die Wiederherstellung der vollkommenen Majestät des Rechts. Die Regierung selbst muß darauf bedacht sein, jede Willkür zu vermeiden, sie muß sich daher einer geordneten Kontrolle durch das Volk unterstellen [... ${ }^{10}{ }^{1}$. Dieses Ziel, an dem sie alle auf unterschiedliche Weise mitwirkten, verband die Gruppe und unterschied sie von ihren Zeitgenossen. Wer darum wusste, war in permanenter Gefahr, entdeckt, des Hochverrates angeklagt und zum Tode verurteilt zu werden. Die bewusste Entscheidung, für das Attentat, den Staatsstreich und den Wiederaufbau eines neuen Staates unter Lebensgefahr tätig zu werden, intensivierte die Beziehungen der Verschwörer untereinander und stellte die Inklusion der Gruppe dar.

Wie stark diese Bindung werden konnte, belegen Erinnerungen der Angehörigen der Beteiligten. Die Tochter von Carl Goerdeler, dem ehemaligen Oberbürgermeister von Leipzig, der nach seinem Rücktritt 1937 zu einer zentralen Person des konservativen bürgerlichen Widerstandes wurde, stellte fest: »[...] die neuen Kontakte bedeuteten ihm mehr als sgute Beziehungen<, waren nicht bloß nützliche Verbindungen zu einflussreichen Persönlichkeiten. Die psychische Belastung im Widerstand verlangte nach seelischem Halt ${ }^{11}$. Und weiter: »Meinem Vater ist es gelungen, kontinuierlich, nicht nur in besonders

8 Nähere Angaben hierzu werden in dem laufenden Dissertationsprojekt der Verfasserin »Eine sganz klein Cliquer? Die nationalsozialistischen Ermittlungen über den Umsturzversuch vom 20. Juli 1944. Eine historische Netzwerkanalyse"zu finden sein, welches von Prof. Dr. Bernhard Kroener (Universität Potsdam) betreut wird.

$9 \mathrm{Zu}$ den Vorstellungen der Beteiligten des 20. Juli 1944 bezüglich eines neu zu schaffenden Rechtsstaates vgl. u. a. Peter Steinbach, Der 20. Juli 1944. Gesichter des Widerstands, München 2004; vgl. hier insbesondere das Kapitel »«Mensch geht vor Prinzip«. Für die Wiederherstellung des Rechts«, S. 283-301.

10 Die Gestapo-Beamten fanden den Entwurf dieser Regierungserklärung in der Hinterlassenschaft von Carl Goerdeler. Sie ist einem Schreiben von Ernst Kaltenbrunner an Martin Bormann vom 5. August 1944 als Anlage beigefügt, siehe Jacobsen, Spiegelbild (wie Anm. 2), S. 147.

11 Marianne Meyer-Krahmer, Carl Goerdeler - Mut zum Widerstand. Eine Tochter erinnert sich, Leipzig 1998, S. 139. 
kritischen Phasen, Gefährten zu finden, die einander ermutigten. Freundschaft bot oft den einzigen Halt bei dem Versuch, sich dem übermächtigen Sog des Geschehens entgegen zu stemmen ${ }^{12}$. "Auch die Tochter von Karl Ludwig Freiherr von Guttenberg, der die monarchistische Zeitschrift »Weiße Blätter« herausgab und ab $1941 \mathrm{im}$ Amt Ausland/Abwehr tätig war, stellte Ähnliches fest: »Wenn sich aber einmal so ein Kreis gebildet hatte [Personen, die Kritisches hören und wahrhaben wollten, d. Verf.], entstanden Freundschaften von einer unbeschreiblichen Intensität. Aus ihnen erwuchs den Verschwörern die Kraft und der Mut zum Handeln, den die meisten ihrer Zeitgenossen nicht aufbrachten. Diese Freundschaften setzten sich über alle Grenzen hinweg, die die Herkunft der einzelnen möglicherweise gezogen hatte ${ }^{13}$.«

Ein verallgemeinernder Rückschluss der eben beschriebenen Beziehungsform auf die hohe Intensität sämtlicher Beziehungen der Verschwörer untereinander würde dennoch zu kurz greifen. Neben engen Freundschaften gab es natürlich auch weniger intensive Beziehungen, auch wird hin und wieder von Befremdung und Distanz oder auch Ablehnung zwischen den Beteiligten gesprochen. So war beispielsweise die Beziehung zwischen Stauffenberg und Goerdeler nicht konfliktfrei. Stauffenberg teilte zwar die Auffassungen Goerdelers zu großen Teilen. Doch zog er den Sozialdemokraten Julius Leber als zukünftigen Reichskanzler vor ${ }^{14}$. Goerdeler beschwerte sich hingegen noch brieflich bei Stauffenberg über seine selbstständigen Verhandlungen mit den Arbeiterführern, was seiner Auffassung nach nicht die Aufgabe der Militärs sei $^{15}$. Ein weiteres Beispiel von Dissonanzen findet sich in den Tagebüchern des ehemaligen deutschen Botschafters in Rom, Ulrich von Hassell. Im Frühjahr 1942 konstatierte er Unstimmigkeiten zwischen Vertretern der konservativen Elite und Angehörigen des Militärs, zwischen denen er zu vermitteln versuchte. Er sprach vom »Kreise Geibel [Beck] usw., wo immer noch, besonders seit der letzten Panne mit Scherz [Witzleben] alles stark auseinanderläuft. Die Panne selbst ist ziemlich repariert, immerhin sind einige Mißtrauensreste noch da (Geißler [Popitz] gegen Hase [Oster], junger Kreis gegen Geißler [Popitz] usw.). Ich bemühe mich um den Ausgleich, gehe heute mit dem Bürger der Barcel[oneser] Handelskammer [Olbricht] zu Geibel [Beck], der zentral die Fäden halten muß $\beta^{16}$ «. Neben diesen, zwischen den einzelnen Verschwörergruppen existierenden Spannungen, gab es selbst innerhalb der Gruppen

Ebd., S. 143.

13 Maria Theodora von dem Bottlenberg-Landsberg, Karl Ludwig Freiherr von und zu Guttenberg. 1902-1945. Ein Lebensbild, Berlin 2003, S. 19.

14 Detlef Graf von Schwerin, »Dann sind «s die besten Köpfe, die man hängt. « Die junge Generation im deutschen Widerstand, München 1991, S. 357.

15 Ebd., S. 367.

16 Ulrich von Hassell, Die Hassell-Tagebücher 1938-44. Aufzeichnungen vom Andern Deutschland, hrsg. von Friedrich Hiller von Gaertringen, 2. Aufl., Berlin 1989, S. 305. Der Tagebucheintrag stammt vom 22.3.1942. Die Auflösungen der Decknamen sind durch den Herausgeber vorgenommen worden. Mit der „Panne“ ist vermutlich gemeint, dass die noch unausgereiften Umsturzvorbereitungen Generalfeldmarschall Erwin von Witzleben mitgeteilt worden sind, der diese als unzureichend empfand. Vgl. ebd., S. 558, Anm. 21. 
Dissonanzen. So bestand wohl eine tiefere Abneigung zwischen Theodor Steltzer und Eugen Gerstenmaier, die beide dem Kreisauer Kreis angehörten ${ }^{17}$.

Trotz inhaltlich oder persönlich begründeten Unstimmigkeiten zwischen und innerhalb der einzelnen Gruppen des Netzwerkes vom 20. Juli 1944 stellte das notwendige Vertrauen, auf das die Beteiligten gegenseitig angewiesen waren, um gemeinsam im Geheimen tätig werden zu können, doch die Grundlage für die Inklusion der Gruppe dar ${ }^{18}$.

\section{Wahrung des Geheimnisses}

Die Geheimhaltung des Umsturzvorhabens war für dessen Erfolg von elementarer Bedeutung. Um dieses Geheimnis wahren zu können, galt es, bestimmte Verhaltensregeln zu berücksichtigen. So wurden nur sehr wenigen Verschwörern konkrete Informationen über den Zeitpunkt und den Ort des Attentates bekannt gegeben. Namen und detailliertere Informationen wurden nur weiter gegeben, wenn es unbedingt notwendig erschien ${ }^{19}$. Telefonate oder schriftliche Belege über konspirative Tätigkeiten wurden wenn möglich vermieden, um nicht abgehört zu werden und um der Gestapo so wenig Beweismaterial wie möglich zu liefern. Durch die notwendige Geheimhaltung waren die Kommunikationsmöglichkeiten der Verschwörer daher stark eingeschränkt. Die sicherste Form stellte das persönliche Gespräch in kleinster Runde dar ${ }^{20}$. Doch da die Verschwörer nicht nur in Berlin und Umgebung wohnten und wirkten, sondern sich das Widerstandsnetz über weite Teile des deutschen Machtbereichs erstreckte, waren diese persönlichen Gespräche auch stets mit erhöhtem

17 Der Name » Kreisauer Kreis« ist ursprünglich eine Bezeichnung der Gestapo, die von der Forschung übernommen worden ist. Der Name nimmt Bezug auf das Moltkesche Familien-Gut Kreisau in Schlesien, wo mehrere Besprechungen stattfanden. Steltzer schrieb über Gerstenmaier: »sehr schwer zu charakterisierender, dynamischer Typ, klug, ehrgeizig, menschlich kontaktarm, nicht sehr beliebt «, siehe Steltzers Charakterisierung der Kreisauer auf Anfrage von Ger van Roon, vom 18.07.1961: Institut für Zeitgeschichte, ZS/A-18, Bd. 7., zit. nach. Klaus Philippi, Die Genese des »Kreisauer Kreises«. Stuttgart 2012, S. 82. Vgl. auch Klaus Alberts, Theodor Steltzer. Szenarien seines Lebens. Eine Biographie, Heide 2009, S. 33.

18 Nähere Angaben hierzu werden in dem Dissertationsprojekt der Verfasserin zu finden sein.

19 Eduard Brücklmeier sagte gegenüber der Gestapo beispielsweise aus: »Einzelheiten hat mir Schwerin nicht gegeben. Dies war für mich nicht besonders erstaunlich, da es schon oft vorgekommen war, daß man mir Einzelheiten nicht sagen wollte mit der Bemerkung, es wäre jeder durch Beck persönlich verpflichtet, nur das zu sagen, was der andere unbedingt wissen müsse." Siehe Jacobsen, Spiegelbild (wie Anm. 2), Schreiben vom 12.12.1944, S. 522. Im gleichen Schreiben heißt es weiter: »Wie weit dieses konspirative System der Geheimhaltung und des aufgeteilten Vertrauens ging, ergibt sich, z. B. aus einer Äußerung Goerdelers, der aussagt, daß auch Beck ihm Namen nur mit großer Zurückhaltung gesagt habe. Geheimhaltung selbst gegenüber nächsten Vertrauten sei diesem alten Generalstäbler zur zweiten Natur geworden. « Siehe ebd.

20 Vgl. u. a. Bodo Scheurig, Henning von Tresckow. Biographie, 3. Aufl., Oldenburg 1973, S. 193: »Tresckow benutzte weder Post noch Telefon. Wände hatten für ihn Ohren. Stauffenberg traf er im Grunewald. Nahezu jede Zusammenkunft erzwang Umwege und war mühsam einzufädeln.« 
logistischem und auch finanziellem Aufwand verbunden. Zudem waren direkte Rückfragen oft nicht immer möglich, und nicht selten kam es dadurch zu Verzögerungen von Planungen und Handlungen. Ebenfalls entstanden Verzögerungen durch logistische Schwierigkeiten oder unvorhersehbare Zwischenfälle. So hatte Otto John beispielsweise eine Verbindung zwischen dem Preußischen Finanzminister Johannes Popitz und Generalfeldmarschall Erwin von Witzleben herzustellen. Aufgrund eines erneuten Kuraufenthaltes Witzlebens und Johns anschließender Reise nach Madrid konnte dies jedoch nicht erfolgen $^{21}$. Und auch bei persönlichen Treffen lauerte stets die Gefahr, beobachtet und verraten zu werden. In seinen Erinnerungen schilderte Hans Bernd Gisevius u. a. die permanente Bedrohung durch die Beschattung zentraler Persönlichkeiten durch die Gestapo: »Ich muß mich beeilen, soll nicht Beck warten. In Lichterfelde schlage ich einen kleinen Umweg ein. Ich möchte von allen Seiten kontrollieren, ob verdächtige Gestalten lauern. Erfreulicherweise ist die nähere Umgebung der Goethestraße so zerbombt, daß es für Spitzel schwer ist, sich unauffällig aufzustellen. Als ich dieses Beck erzähle, lächelt er verschmitzt. [...] Eine Bombe habe das Nachbarhaus getroffen. [...] Das Eckzimmer dieses nun in Trümmern liegenden Hauses war von der Gestapo beschlagnahmt, um Becks Verkehr zu beobachten und zu fotografieren ${ }^{22}$ «.

Da große konspirative Treffen aus Sicherheitsgründen vermieden wurden, waren viele Verschwörer untereinander nicht bekannt. In der Regel trafen sich zwei oder drei Personen, um sich auszutauschen und um an den Planungen weiterzuarbeiten. Beispielhaft dargestellt werden diese Einzeltreffen in den Briefen von Helmuth James Graf von Moltke an seine Frau Freya. Am 3. Juli 1941 schrieb er: »Heute Mittag aß ich mit einem Freund von Lukaschek, abends gehe ich zu Yorcks und nachts nach Nikolassee. Freitag Mittag essen Yorck \& Haeften in der Derfflingerstr., um 3.30 trinken Yorck \& Einsiedel bei mir Thee und um 6 kommt Mierendorff. Also ein typischer Großkampftag. Sonnabend Mittag esse ich bei Trotts, Sonntag Mittag bei Üx, nachmittags bei Kieps - [...] Da hast Du mein Wochenprogramm ${ }^{23}$. "Deutlich wird hier, dass sich Moltke mit den Mitverschwörern fast immer einzeln traf, auch wenn dies nicht nur logistische Schwierigkeiten mit sich brachte, sondern darüber hinaus auch sehr zeitaufwändig war. Für die Sicherheit der Gruppe schien dieses Vorgehen jedoch geboten. Insgesamt gab es nur drei größere Treffen in Kreisau auf dem Gut der Familie von Moltke in Schlesien ${ }^{24}$. Doch auch hier waren nie alle der rund 20 dem Kreisauer Kreis zuzurechnenden Personen zeitgleich vertreten.

${ }_{21}$ Vgl. Peter Hoffmann, Widerstand, Staatsstreich, Attentat. Der Kampf der Opposition gegen Hitler, 4. Aufl., München 1985, S. 449.

22 Hans Bernd Gisevius, Bis zum bitteren Ende. Vom Reichstagsbrand bis zum 20. Juli 1944, Hamburg 1960, S. 482 f.

23 Helmuth James von Moltke, Briefe an Freya 1939-1945, hrsg. von Beate Ruhm von Oppen, München 2007, S. 260. Bei den Genannten handelt es sich um Hans Lukascheck, Peter Graf Yorck von Wartenberg und seine Frau Marion, Hans Werner von Haeften, Horst von Einsiedel, Carlo Mierendorff, Adam von Trott zu Solz und seine Frau Clarita sowie Nikolaus Graf von Üxküll-Gyllenband (Üx).

24 Die erste Tagung in Kreisau fand vom 22.-25. Mai 1942 statt, die zweite vom 16.-18. Oktober 1942, die dritte vom 12.-14. Juni 1943. 
Daher waren nicht einmal alle Angehörigen dieser »Clique« des Gesamtnetzwerkes vom 20. Juli 1944 miteinander bekannt.

Eine der wenigen größeren Besprechungen, auf der zwischen verschiedenen Interessensgruppen vermittelt wurde, fand im Januar 1943 statt - als die Niederlage von Stalingrad schon offensichtlich geworden war. An diesem besonders von Fritz-Dietlof Graf von der Schulenburg ausgehenden Treffen bei Peter Graf Yorck von Wartenburg nahmen Angehörige des Kreisauer Kreises auf der einen und Vertreter der alten konservativen Elite auf der anderen Seite teil. Bis auf Moltke waren sich zumindest alle einig, dass der Staatsstreich bald erfolgen müsse. Meinungsverschiedenheiten wurden hingegen vor allem bezüglich sozial- und wirtschaftspolitischer Fragen deutlich ${ }^{25}$. Diese großen Treffen blieben jedoch eine Ausnahme, die meisten Gespräche fanden in kleinem Rahmen statt. So hat auch Wilhelm Leuschner, der kontinuierlich um die Gewinnung weiterer Unterstützer aus Arbeiter- und Gewerkschaftskreisen für den Umsturz bemüht war, zahlreiche persönliche Gespräche im gesamten Reichsgebiet geführt ${ }^{26}$.

Zum Schutz der Gruppe und des Geheimnisses galten darüber hinaus noch verschiedene weitere Regeln. Grundsätzlich versuchten die Beteiligten, sämtliche konspirativen Kontakte durch dienstliche Verbindungen zu decken und diese somit gegenüber der Gestapo jederzeit beruflich begründen zu können. Hierfür war jedoch häufig die Besetzung von Schlüsselpositionen in militärischen Einheiten und in zivilen Dienststellen und Unternehmen von Bedeutung. Dies führte nicht selten zu einem komplizierten und auch verwirrenden Doppelspiel. Um bestimmte Positionen besetzt halten zu können, traten einige Mitverschwörer zur Tarnung in die Partei oder andere NS-Organisationen ein. Andere wurden durch die Ausführung ihrer Ämter und Aufgaben mitschuldig an der Fortführung des Krieges und teilweise auch an NS-Verbrechen $^{27}$. Wie sehr sich einige Verschwörer ihrer eigenen Schuld und Verstrickung in das NS-Regime bewusst waren, belegen verschiedene Selbstzeugnisse. So schrieb beispielsweise der Chef der Abteilung Fremde Heere West im Oberkommando des Heeres, Oberst i.G. Alexis Freiherr von Roenne, der als Mitwisser angeklagt und schließlich zum Tode verurteilt wurde, am 1. Oktober 1944 auf einem Kassiber aus der Haft an seine Frau: »Er [Gott, d. Verf.] hat vielleicht auch dem deutschen Volk die im Gelingen des Umsturzes vielleicht liegende Rettungsmöglichkeit vor dem Verderben nicht mehr schenken wollen, um seiner schwersten Schuld willen (Antichristentum, Judenmassaker etc.), die es nun nach Jahren vergeblicher Gnade büßen soll!« In einem zweiten, undatierten Kassiber heißt es: »Ich weiß um Dein verzweifeltes Flehen, Deine ganze arme Zerrissenheit! Aber wir müssen uns demütig

25 Schwerin, Köpfe (wie Anm. 14), S. 271 f.

26 Vgl. Axel Ulrich, Wilhelm Leuschner - Ein deutscher Widerstandskämpfer. Für Freiheit und Recht, Einheit der Demokraten und eine soziale Republik, Wiesbaden 2012, S. 51, 62.

27 Vgl. u. a. Gerd R. Ueberschär, NS-Verbrechen und der militärische Widerstand gegen Hitler, Darmstadt 2000. Zur Komplexität des Themas vgl. u. a. Winfried Heinemann, Kriegführung und militärischer Widerstand im Bereich der Heeresgruppe Mitte an der Ostfront. In: Ebd., S. 77-89. 
und mitschuldig in einem unvorstellbar frevelhaftem Volk sehen und uns nun mit unter sein Gericht beugen, das bald so unendlich viele erfassen wird ${ }^{28}$.«

Neben den bereits genannten Tarnungsbemühungen wurden häufig auch Decknamen zur besseren Geheimhaltung und zum Schutz der Beteiligten verwendet, wie bereits durch den oben zitierten Tagebucheintrag Hassells vom 22. März 1942 deutlich wurde. Die Tarnnamen, die mitunter auf einen thematischen Bezug, ein Wortspiel oder eine persönliche Erinnerung verwiesen, wurden jedoch keineswegs einheitlich verwendet, wie ein kurzer Vergleich der Tagebücher Hassells mit den Aufzeichnungen des als Reserveoffizier im Allgemeinen Heeresamt tätigen Studienrats Hermann Kaiser zeigen kann. Carl Langbehn erhielt durch Hassell den zunächst leicht zu entschlüsselnden Decknamen »Kurzfuß «, später den besser getarnten Namen »Walcher«. Letzterer entstand in Anlehnung an Langbehns Haus am Walchensee, in dem ihn Hassell einige Male aufgesucht hatte. Für Hans Oster fand Hassell den Tarnnamen »Hase«, für Friedrich Olbricht die schwerer zu dekodierende Bezeichnung »Baceloneser Pfarrer« bzw. »Bürger der Barcel. Handelskammer«, in Erinnerung an einen ihm bekannten Namensvetter Olbrichts in Barcelona ${ }^{29}$. Auch Hermann Kaiser nutzte eigens erdachte Wortspiele wie »Dunkelstadt « für Wolf Heinrich Graf von Helldorf. Anders als bei Hassell ist Olbricht bei Kaiser unter dem Tarnnamen »Schwimmer « oder »O I « zu finden, Oster hingegen unter $\gg \mathrm{O} I \mathrm{I} \ll$ bzw. $»$ Frühling $^{30}$.

Neben der Verwendung von Decknamen wurde zudem versucht, so wenig riskante Aktionen zu unternehmen wie möglich, um nicht die Aufmerksamkeit von NS-Behörden, Spitzeln oder Denunzianten auf sich zu ziehen. Wilhelm Leuschner hielt daher auch viele seiner Verbindungsleute an, auf riskante Flugblatt- oder ähnliche Widerstandsaktionen zu verzichten, um das eigentliche Vorhaben auf diese Weise nicht unnötig zu gefährden ${ }^{31}$. Auch wurde sehr darauf geachtet, jeglichen Kontakt mit kommunistischen Gruppen zu vermeiden. War eine inhaltliche Zusammenarbeit mit den Kommunisten für fast alle Mitverschwörer ohnehin undenkbar, traten sicherheitsbedingte Überlegungen noch hinzu, da der kommunistische Widerstand seit 1933 von der Gestapo systematisch verfolgt und mit Spitzeln durchsetzt worden war. Als sich Julius Leber und Adolf Reichwein am 22. Juni 1944 doch noch zu einer Kontaktaufnahme mit Kommunisten entschieden, um gemeinsam gegen die Nationalso-

28 Die Familie von Alexis Freiherr von Roenne hat seinen Nachlass 2015 als Schenkung ans Militärhistorische Museum übergeben. Die erwähnten Kassiber sind in der Dauerausstellung des Militärhistorischen Museums in Dresden zu sehen (MHM, BBAT4501 und BBAT4502. Zur ausführlichen Kommentierung der Kassiber siehe: Magnus Pahl, Motive und Ziele - Geheime Aufzeichnungen von Oberst i.G. Alexis Freiherr von Roenne, in: Attentat auf Hitler. Stauffenberg und mehr (Katalog zur Ausstellung im Militärhistorischen Museum der Bundeswehr vom 4.7.-4.11.2014), hrsg. v. Linda von Keyserlingk, Gorch Pieken und Matthias Rogg, Dresden 2014, S. 38-47.

29 Zur Hassells Verwendung der Decknamen vgl. Hassell-Tagebücher (wie Anm. 16), S. 42 f.

30 Mut zum Bekenntnis: Die geheimen Tagebücher des Hauptmanns Hermann Kaiser 1941/1943, hrsg. von Peter M. Kaiser, Berlin 2010. Zur Verwendung und Aufschlüsselung der durch Kaiser benutzten Decknamen vgl. insbes. das Vorwort in ebd., S. 39.

31 Vgl. Ulrich, Wilhelm Leuschner (wie Anm. 26), S. 56. 
zialisten vorgehen zu können, erwiesen sich diese Befürchtungen als berechtigt. Beide Verschwörer wurden Anfang Juli 1944 verhaftet, da an dem Gespräch mit den kommunistischen Widerstandskämpfern Anton Saefkow und Franz Jacob auch ein Spitzel teilgenommen hatte ${ }^{32}$.

\section{Vermittlung und Rekrutierung}

In regimekritischen Kreisen begann bereits ab 1933/34 ein noch nicht zielgerichtetes Kontakteknüpfen von Unzufriedenen und Besorgten. So schreibt Peter Hoffmann: »Es bedurfte der vier Jahre von 1934 bis 1938, um die Gegner des Nationalsozialismus aus Gewissensgründen von den Mittuenden, Gleichgültigen und Unentschlossenen allmählich zu sondern ${ }^{33}$. «Besonders früh begannen Gewerkschafter und Sozialdemokraten, deren Organisationsstrukturen bereits 1933 verboten und zerschlagen worden waren, ein reichsweites Netzwerk aus Regimekritikern aufzubauen ${ }^{34}$. Die konservativen Regimegegner wurden in der Regel erst in der zweiten Hälfte der 1930er Jahre aktiv. Detlef von Schwerin zeichnet für die Jahre 1936 bis 1938 für den Freundes- und Bekanntenkreis seines Vaters ein Bild von einer sich ganz bewusst orientierenden und handelnden Gruppe in einer Anfangsphase des aktiven Widerstandes: »All das Hin und Her, Fädenziehen, Kontaktknüpfen stand jetzt schon im Zeichen des Widerstandes. Sie wussten noch nicht, wann und wie sie all diese vielfältigen Beziehungen einsetzen würden, aber sie bereiteten sich vor. Sie waren dabei im Staatsgefüge so platziert, dass ihnen nichts Wesentliches entgehen konnte: in der Abwehr, im Berliner Polizeipräsidium, im Auswärtigen Amt und in der Dienststelle Ribbentrop ${ }^{35}$.«

Erste Anzeichen von systematischem regimekritischem Verhalten auf militärischer Seite sind ab 1936/37 im Umkreis des militärischen Nachrichtendienstes um den damaligen Oberstleutnant Hans Oster zu finden. Jedoch handelte es sich dabei eher um einen von seinem Vorgesetzten Admiral »Canaris geduldeten und geförderten innerpolitischen Informations- und Nachrichtendienst $\ll^{36}$, mit dem besonders Verbrechen von SS und SD aufgedeckt werden sollten. Hierfür nutzte Oster »eine Art von sehr lockerem >OldBoy-Network< ehemaliger Freikorpsler und Rechtskonservativer, ergänzt durch zufällige oder gesellschaftlich etablierte Verbindungen mit kritisch eingestellten Einzelpersönlichkeiten $\ll^{37}$. Erst 1938 begann Hans Oster, gemein-

32 Vgl. hierzu u. a. Schwerin, Köpfe (wie Anm. 14), S. 371.

33 Hoffmann, Widerstand (wie Anm. 21), S. 45; vgl. auch Otto John, »Falsch und zu spät«. Der 20. Juli 1944. Epilog, München, Berlin 1989, S. 108 f.

34 Für den bereits 1933/34 einsetzenden Aufbau eines sozialdemokratischen, gewerkschaftlichen Netzwerkes vgl. Ulrich, Wilhelm Leuschner (wie Anm. 26), S. 51.

35 Schwerin, Köpfe (wie Anm. 14), S. 138.

36 Klaus-Jürgen Müller, Struktur und Entwicklung (wie Anm. 7), S. 99.

37 Ebd., S. 99. 
sam mit einigen seiner Mitarbeiter sowie anderen zivilen und militärischen Vertrauten konkrete Staatsstreichpläne zu entwickeln. Zu Beginn dieses Jahres war der Oberbefehlshaber des Heeres, Generaloberst Werner von Fritsch, durch eine Intrige ehrlos entlassen worden. Vergeblich hatten der Chef des Generalstabes des Heeres, General der Artillerie Ludwig Beck, Oberstleutnant Hans Oster und einige Gleichgesinnte versucht, die Generalität daraufhin zu einem gemeinsamen Protest zu motivieren. Die Sudetenkrise und die damit ständig wachsende Kriegsgefahr führten im Sommer und Herbst 1938 zu konspirativen Verbindungen zwischen dem Generalstab des Heeres, der Abwehr und dem Auswärtigen Amt und schließlich zu gemeinsamen Bemühungen, den Krieg zu verhindern ${ }^{38}$. In den Septemberwochen 1938 vor und während dem Münchner Abkommen sollte es tatsächlich zu relativ gut vorbereiteten Umsturzbemühungen kommen ${ }^{39}$. Ob dieser beinahe ausgelöste Staatsstreichversuch ausschließlich am Zustandekommen des Münchener Abkommens scheiterte, oder ob er auch ohne das Nachgeben der Westmächte erfolglos geblieben wäre, ist umstritten ${ }^{40}$. Festzustellen ist jedoch, dass diese Entwicklungen den Aufbau eines konspirativen Netzes auch in militärischen Kreisen wesentlich befördert haben.

Entscheidend für die Auswahl neuer Mitverschwörer war die persönliche Einstellung gegenüber dem NS-Regime, die Bereitschaft zum Handeln sowie die Position der jeweiligen Person und deren Handlungsspielraum. Um das Jahr 1938 fallen auch die ersten explizit für einen Umsturzversuch vermittelten Kontakte. So sollen sich Oster und Leuschner nach der Fritsch-Krise Anfang 1938 über Friedrich Wilhelm Heinz kennen gelernt haben ${ }^{41}$. Der Jurist Josef Wirmer stellte schließlich im Frühherbst 1939 eine ständige Verbindung der Gruppe Kaiser-Leuschner-Habermann zu der Gruppe um Oster her ${ }^{42}$. Da Leuschners Firma kriegswichtige Patente zur Aluminiumverarbeitung besaß, konnte er in den folgenden Jahren zu verschiedenen Mitgliedern des militärischen Widerstands relativ unauffällig Kontakt pflegen ${ }^{43}$. Unter den Personen, welche die unterschiedlichen Gruppen und Persönlichkeiten miteinander in Verbindung brachten, um eine breitere und effektivere Basis für den Wider-

38 Vgl. u. a. ebd., S. 102. Vgl. auch Romedio G. Thun-Hohenstein, Der Verschwörer. General Oster und die Militäropposition, Berlin 1982, S. 81-118. Zu den unterschiedlichen Zielen der beteiligten Gruppen vgl. ebd., S. 107 f.

39 Vgl. Wolfgang Schieder, Zwei Generationen im militärischen Widerstand gegen Hitler. In: Widerstand, hrsg. von Schmädeke/Steinbach (wie Anm. 5), S. 436-459, S. 443 f; Helmut Krausnick, Vorgeschichte und Beginn des militärischen Widerstandes gegen Hitler. In: Vollmacht des Gewissens, Bd. 1, München 1956, S. 336-365; Hoffmann, Widerstand (wie Anm. 21), S. 109-129.

40 Schieder vertritt die Ansicht, dass der Staatsstreich »in allen Fällen gescheitert wäre« und schließt sich damit Eberhard Jäckel an. Vgl. Schieder, Zwei Generationen (wie Anm. 41), S. 444 bzw. Eberhard Jäckel, Wenn der Anschlag gelungen wäre. In: Der Zwanzigste Juli, Alternative zu Hitler?, hrsg. von Hans Jürgen Schultz, Stuttgart u. a. 1974, S. 69-76. Die Gegenposition ist u. a. zu finden bei Ger van Roon, Widerstand im Dritten Reich. Ein Überblick, 7. Aufl., München 1981, S. 129.

${ }_{41}$ Vgl. Schwerin, Köpfe (wie Anm. 14), S. 353; vgl. auch Hoffmann, Widerstand (wie Anm. 21), S. 124.

42 Vgl. Hoffmann, Widerstand (wie Anm. 21), S. 135.

43 Vgl. Ulrich, Wilhelm Leuschner (wie Anm. 26), S. 42. 
stand zu schaffen, ist neben Carl Goerdeler, Wilhelm Leuschner unter anderen auch Fritz-Dietlof von der Schulenburg zu nennen ${ }^{44}$.

\section{Struktur des Netzwerkes}

Immer wieder wurde in der Forschung auf die Struktur der Widerstandsbewegung verwiesen, wobei besonders Klaus-Jürgen Müller die Bedeutung und die Möglichkeiten einer Strukturanalyse des Widerstandes betont hat ${ }^{45}$. Mithilfe des strukturanalytischen Ansatzes ließe sich, so Müller, beispielsweise feststellen, dass die verschiedenen Interessen der Akteure von 1938 mitunter in Zusammenhang mit den jeweiligen Positionen in einer dienstlichen Hierarchie gestanden hätten. Während hohe Staatsfunktionäre und höchste Militärs wie Ludwig Beck, Franz Halder, Wilhelm Canaris und Ernst von Weizsäcker lediglich einen Krieg unter den gegebenen Umständen verhindern wollten, hätten die unteren Dienstebenen mit Vertretern wie Hans Oster oder Hans Bernd Gisevius, die Kriegsverhinderungsbemühungen lediglich als Instrument für einen innenpolitischen Umsturz betrachtet ${ }^{46}$. Um weitere Rückschlüsse in Bezug auf den Zusammenhang zwischen Strukturmerkmalen und Motiven, Zielen sowie Eigenarten des Widerstandes ziehen zu können, erscheint eine umfangreiche genauere Untersuchung der Struktur der beteiligten Personengruppen notwendig, die im Rahmen dieses Beitrages jedoch nicht geleistet werden kann ${ }^{47}$. Dennoch sollen im Folgenden auf netzwerkanalytischer Grundlage einige Strukturmerkmale dargestellt werden.

Die innere Struktur des Netzwerkes vom 20. Juli 1944 ist bisher auf unterschiedliche Weise beschrieben worden. Mehrere Mitverschwörer haben von verschiedenen Widerstandskreisen gesprochen, die sich teilweise überlagerten. Fabian von Schlabrendorff sprach nach 1945 beispielsweise von einer »Vielfalt von Kreisen«, »die sich gegenseitig überschnitten, wobei bald der eine im Mittelpunkt stand, bald der andere $\ll^{48}$. Auch Theodor Steltzer beschrieb nach dem Krieg verschiedene Kreise, die sich für Widerstandszwecke einander annäher-

${ }^{44}$ Vgl. Ulrich Heinemann, Ein konservativer Rebell. Fritz-Dietlof Graf von der Schulenburg und der 20. Juli. Berlin 1990; vgl. auch Linda von Keyserlingk, Der 20. Juli 1944 war nicht die Tat eines Einzelnen. Das Netzwerk des Widerstands um Fritz-Dietlof Graf von der Schulenburg, Berlin 2009.

45 Vgl. Müller, Struktur und Eigenart (wie Anm. 5), S. 89-133 sowie Müller, Struktur und Entwicklung (wie Anm. 7), S. 329-344; vgl. auch Gerd R. Ueberschär, Militäropposition gegen Hitlers Kriegspolitik 1939 bis 1941. Motive, Struktur und Alternativvorstellungen des entstehenden militärischen Widerstands. In: Widerstand, hrsg. von Schmädeke/Steinbach (wie Anm. 5), S. 345-367.

46 Müller, Struktur und Entwicklung (wie Anm. 7), S. 108 f.

47 Eine solche genaue Strukturanalyse wird in dem laufenden Dissertationsprojekt der Verfasserin vorgenommen.

48 Fabian von Schlabrendorff, Offiziere gegen Hitler, Berlin 1984, S. 27. 
ten ${ }^{49}$. Einige Historiker bevorzugten die Bezeichnung »Gruppe«, beschrieben letztendlich aber die gleichen Strukturmerkmale ${ }^{50}$.

Fritz-Dietlof von der Schulenburg hat laut den Gestapo-Verhören im Dezember 1944 hingegen von vier ineinander verschachtelten Kreisen der Widerstandsbewegung um den 20. Juli gesprochen. Zum ersten, innersten Kreis, in dem beispielsweise die Sprengstofffrage besprochen wurde, hätten nur sehr wenige gehört. Ein etwas größerer Kreis sei in die Attentatspläne eingeweiht gewesen, ein noch größerer Kreis sei darüber informiert gewesen, dass ein gewaltsames Unternehmen geplant sei, und schließlich habe es sehr viele Personen gegeben, mit denen die Eingeweihten recht allgemein über den Ernst der Lage gesprochen hätten ${ }^{51}$.

Die sich auf den ersten Blick widersprechenden Beschreibungen lassen sich jedoch auf den zweiten Blick miteinander verbinden, wenn die Gesamtstruktur nicht als Summe einzelner Kreise oder Gruppen, sondern als dynamisches Netzwerk mit komplexen Strukturen begriffen wird. Die einzelnen Gruppen des Widerstandes können aus netzwerkanalytischer Sicht als »Cliquen « bezeichnet werden, die in sich stark vernetzt waren und mit anderen Gruppen in Kontakt standen, jedoch hier eine weniger hohe Kontaktdichte aufwiesen. Bereits bei einer oberflächlichen Durchsicht der Primärquellen (Briefe, Tagebücher, Gestapo-Verhörprotokolle u. ä.) und Erinnerungsberichten als Sekundärquellen wird deutlich, dass es im Netzwerk des 20. Juli unzählige Überschneidungen zwischen den Gruppen und damit keine scharfen Trennlinien zwischen den einzelnen zivilen und auch militärischen »Cliquen « gab. Innerhalb von Letzteren gab es jedoch Personen, die stärker oder weniger stark in das Gesamtnetzwerk eingebunden und sowohl im Hinblick auf die Anzahl der Kontakte wie auch in Bezug auf die konkrete Staatsstreichplanung stärker mit den zentralen Personen der Verschwörung verbunden waren. Diese unterschiedliche Zentralität ${ }^{52}$ der Personen unabhängig ihrer Zugehörigkeit zu einer

49 Theodor Steltzer, Sechzig Jahre Zeitgenosse, München 1966, S. 153: »So standen wir mit dem Kreis der Generale Beck, Olbricht und von Witzleben in Verbindung, in dem später Graf Stauffenberg die Führung an sich riss. Ohne seine Initiative wäre es kaum zu einem 20. Juli gekommen. Dann gab es den Gewerkschafts- und SPD-Kreis um Leuschner, zu dem Mierendorff, Haubach, Leber und Maß gehörten. Endlich möchte ich noch die Kreise um Goerdeler und Popitz erwähnen. Neben unseren grundsätzlichen Meinungsverschiedenheiten verhinderten natürlich Terror und Bespitzelung eine umfassendere Zusammenarbeit, die in der Frage eines praktischen Vorgehens eine Annäherung herbeigeführt hätte.«

50 Vgl. Tyrannenmord. Der 20. Juli 1944 und Österreich. Heeresgeschichtliches Museum, hrsg. von Manfried Rauchensteiner, Wien 2004, S. 14: »Außer der schon relativ großen Gruppe von Oppositionskräften in Berlin existierten 1943 ähnliche Gruppen in Paris, in Brüssel und bei der Heeresgruppe Mitte, und nach und nach griff die Organisation auf die meisten Wehrkreise über - so auch auf Wien. Dabei erfuhr die Militäropposition ihre endgültige Strukturierung.« Vgl. auch Detlef Graf von Schwerin, Der Weg der >Jungen Generation< in den Widerstand. In: Widerstand, hrsg. von Schmädeke/Steinbach (wie Anm. 5), S. 462: »Will man die Entstehungsgeschichte des Widerstandes begreifen, seine Mechanik verstehen, muss man den verschiedensten Freundeszirkeln und -grüppchen nachgehen.«

51 Siehe Jacobsen, Spiegelbild (wie Anm. 2), S. 521. Vgl. auch ebd., S. 49.

52 In der Netzwerkanalyse werden verschiedene Arten von Zentralität unterschieden. Neben der Berechnung der Anzahl der vorhandenen Kontakte geht es unter anderem auch um die Frage von Besetzung strategischer Positionen. Weitere Angaben hierzu finden sich u. a. in Dorothea 
bestimmten »Clique «, kann als eine Struktur von konzentrischen Kreisen skizziert werden, wie Schulenburg es bereits 1944 beschrieben hatte. Die zunächst gegensätzlich erscheinenden Beschreibungen des Netzwerkes von nebeneinander existierenden bzw. sich überlappenden Gruppen auf der einen Seite und Kreisen, die ineinander geschachtelt über ein gemeinsames Zentrum und eine Peripherie verfügen, auf der anderen Seite, können sich daher ergänzen.

Dass es für die Vorbereitung des Umsturzes einer gewissen Koordination und damit auch einer Zentralisation innerhalb des Netzwerkes bedurfte, ist offensichtlich. Das Anwerben neuer Personen, das Kontaktieren ausländischer Verbindungsleute oder die Planung der Abläufe am Tag X mussten aufeinander abgestimmt werden. Ab 1942 liefen die Kommunikationswege bei Generaloberst a.D. Ludwig Beck zusammen, der in engem Austausch mit militärischen und zivilen Kreisen stand. Doch obwohl eine gewisse Zentralisation notwendig war, gab es keine strenge hierarchische Struktur innerhalb des Widerstandes vom 20. Juli 1944. Auch Wilhelm Leuschner, der unermüdlich darum bemüht war, zahlreiche Verbindungsleute unter den ehemaligen Gewerkschaftern zu gewinnen, die zu gegebener Zeit nach einem Umsturz den neuen Staatsaufbau und die Gründung einer Einheitsgewerkschaft stützen sollten, lehnte eine systematische und hierarchisch gegliederte Organisationsstruktur, nicht zuletzt aus Sicherheitsgründen, $a b^{53}$. Die Verfolgung von kommunistischen Widerstandsgruppen hatte gezeigt, dass hierarchische Organisationsstrukturen leichter von der Gestapo aufgedeckt werden konnten als andere ${ }^{54}$.

Auch wenn die Verschwörer daher darauf Wert legten, sich nicht hierarchisch zu organisieren, waren bestimmte Teilgruppen des Netzwerkes dennoch äußeren hierarchischen Strukturen unterworfen. Das militärinterne Netzwerk war in seiner Struktur und Funktionalität im Gegensatz zu dem Beziehungsgeflecht der zivilen Gruppen viel stärker durch derartige äußere Umstände geprägt. Es ist anzunehmen, dass der bestehende streng hierarchische Aufbau, der auf die Person Adolf Hitler geleistete Eid sowie das Prinzip von Befehl und Gehorsam den tatsächlichen und bzw. oder den empfundenen Handlungsspielraum der Soldaten stärker eingrenzte, als dies bei Zivilisten der Fall gewesen sein mag.

Der hierarchisch gegliederte militärische Apparat verursachte beispielsweise für die aktiven Verschwörer besondere Schwierigkeiten bei der Planung und Durchführung eines Umsturzes. Die zu einem Umsturz bereiten Militärs be-

Jansen, Einführung in die Netzwerkanalyse. Grundlagen, Methoden, Forschungsbeispiele, 3. Aufl., Wiesbaden 2006, S. 127-162.

53 Vgl. Ulrich, Wilhelm Leuschner (wie Anm. 26), S. 65.

54 Ebd., S. 51: "Zug um Zug mussten weitere Mitstreiter gewonnen werden, die in der Stunde X aktiviert werden könnten. Natürlich dürfte es keine Kaderorganisation im herkömmlichen Sinne sein, vor allem keine starre, straff durchorganisierte Widerstandsstruktur wie die der Kommunisten, deren illegale Verbindungen immer wieder leicht aufgerollt werden konnten, sobald den faschistischen Fahndern ein erster Einbruch in deren auch keineswegs sonderlich professionell getarnten konspirativen Apparat gelungen war.«. Zur Struktur kommunistischer Netzwerke und den Ermittlungsstrategien der Gestapo vgl. u. a. Ulrich Eumann, Das Netz des Siegfried Bittermann. Eine explorative Netzwerkanalyse des Widerstands. In: Jahrbuch für Historische Kommunismusforschung 1 (2012), S. 323-340. 
fanden sich in bestimmten Abhängigkeitsstrukturen, die ihre Handlungsspielräume einschränkten ${ }^{55}$. Für das Gelingen des Staatsstreiches erschien es notwendig, auch hohe Militärs, insbesondere Schlüsselpersonen wie den Chef des Generalstabes des Heeres, Oberbefehlshaber der Heeresgruppen, Wehrmachtbefehlshaber und Militärbefehlshaber in den besetzten Gebieten sowie die Chefs bestimmter, dem OKH unterstellter Dienststellen für die Verschwörung zu gewinnen. Während einige dieser hohen Militärs ihre Unterstützung zusagten, wie der Militärbefehlshaber in Frankreich, General der Infanterie CarlHeinrich von Stülpnagel, oder der Chef des Wehrmachtnachrichten-Verbindungswesen, General der Nachrichtentruppe Erich Fellgiebel, lehnten andere wie beispielsweise Generalfeldmarschall Erich von Manstein, Oberbefehlshaber der Heeresgruppe Don (ab Februar 1943 Heeresgruppe Süd), trotz mehrmaligen Werbungsversuchen eine Beteiligung am Umsturz $a b^{56}$. Andere mochten zwar die Verschwörer grundsätzlich unterstützen, konnten sich jedoch nicht zu einer aktiven Teilnahme durchringen und entschieden sich nach dem gescheiterten Attentatsversuch vom 20. Juli 1944 gegen die Unterstützung des folgenden Staatsstreichversuches. Als prominente Beispiele sind hier Generalfeldmarschall Günther von Kluge als Oberbefehlshaber der Heeresgruppe Mitte bzw. ab Anfang Juli 1944 Oberbefehlshaber West sowie Generaloberst Friedrich Fromm als Chef der Heeresrüstung und Befehlshaber des Ersatzheeres zu nennen. Die Verschwörer sahen sich somit hierarchischen Strukturen unterworfen, die außerhalb des geheimen Netzwerkes waren und dennoch stark auf die Funktionalität und Effektivität dieses Netzwerkes wirkten ${ }^{57}$.

Bei den Versuchen, militärische Gesinnungsgenossen zu finden, stellten die Verschwörer immer wieder fest, dass dem auf die Person Hitler geschworenen Eid auch 1944 noch eine große Bedeutung beizumessen war. Nach dem Tod von Reichspräsident Paul von Hindenburg wurden ab dem 2. August 1934 alle Soldaten auf die Person Hitler vereidigt - unabhängig davon, welchen Eid sie zuvor geleistet hatten ${ }^{58}$. Die Mehrheit der Wehrmachtssoldaten schien nicht

55 Vgl. Ueberschär, Militäropposition (wie Anm. 47), S. 360.

56 Im Februar 1943 wurde Georg Schulze-Büttger als Verbindungsmann zur Heeresgruppe Don versetzt, um Manstein für den Widerstand zu gewinnen. Vgl. Scheurig, Tresckow (wie Anm. 20), S. 155; vgl. auch Jacobsen, Spiegelbild (wie Anm. 2), S. 378. Auch andere Werbungsversuche blieben erfolglos. Vgl. die vergeblichen Versuche Tresckows, seinen Oberbefehlshaber Generalfeldmarschall Feder von Bock oder Generaloberst Heinz Guderian zu gewinnen. Vgl. Peter Hoffmann, Claus Schenk Graf von Stauffenberg. Die Biographie, München 2007, S. 317; vgl. auch Schlabrendorff, Offiziere (wie Anm. 50), S. 100.

57 Zur Person von Friedrich Fromm und zu dessen Beurteilung im Hinblick auf seine Rolle im Widerstand vgl. die umfangreiche Biographie: Bernhard R. Kroener, Generaloberst Friedrich Fromm. Der starke Mann im Heimatkriegsgebiet. Eine Biographie, Paderborn u. a. 2005.

58 Gemäß der Verordnung vom 14. August 1919 hatten die Soldaten der Reichswehr folgende Eidesformel gesprochen: »Ich schwöre Treue der Reichsverfassung und gelobe, dass ich als tapferer Soldat das Deutsche Reich und seine gesetzmäßigen Einrichtungen jederzeit schützen, dem Reichspräsidenten und meinen Vorgesetzten Gehorsam leisten will.« Ab dem 2. August 1934 lautete die Eidesformel: »Ich schwöre bei Gott diesen heiligen Eid, dass ich dem Führer des Deutschen Reiches und Volkes Adolf Hitler, dem Oberbefehlshaber der Wehrmacht, unbedingten Gehorsam leisten und als tapferer Soldat bereit sein will, jederzeit für diesen Eid mein Leben einzusetzen.«Zit. nach: Wolfgang Graf von Vitzthum, Stauffenberg. Zur Rechtfertigung 
zuletzt auch dadurch hinter Hitler und dem NS-Regime zu stehen. Dieser oft auch von hohen Militärs bis zuletzt als bindend empfundene und kaum hinterfragte Eid stellte damit ein strukturelles Problem für die Verschwörung dar. Solange Hitler lebte, zögerten selbst regimekritische Soldaten, sich gegen ihn zu erheben. Um möglichst viele schwankende Generäle noch am Tag des Umsturzes für ihr Vorhaben zu gewinnen, begannen die Verschwörer daher das erste Fernschreiben der >Walküre<-Befehle mit dem Satz »Der Führer Adolf Hitler ist tot.«, um alle Soldaten von ihrem personengebundenen Schwur lösen zu können ${ }^{59}$. Als jedoch bekannt wurde, dass Hitler das Attentat überlebt hatte, funktionierte das von den Verschwörern vorgesehene Befehlssystem nicht mehr ${ }^{60}$.

Auch nach dem Krieg ist noch heftig darüber gestritten worden, wann und warum ein Eidbruch unter rechtlichen sowie unter moralisch-theologischen Gesichtspunkten gerechtfertigt sein könne und unter welchen Umständen er als Hochverrat zu bezeichnen sei. Bei einer rechtlichen Fragestellung spielen das Völkerrecht, das individuelle Widerstandsrecht oder auch fundamentale zivilisatorische Normen eine Rolle. Der Völkerrechtler Wolfgang Vitzthum kam in einem 2011 veröffentlichten Aufsatz zu dem Schluss, dass der Soldateneid von 1934 nur scheinlegal war, da er einen Rechtsbruch darstelle ${ }^{61}$. Eine Rückkehr zum Rechtsstaat sei ohne Staatsstreich nicht mehr möglich gewesen.

\section{Fazit}

Für die Beteiligten des Umsturzversuches vom 20. Juli 1944 war die Geheimhaltung der gemeinsamen Staatsstreichpläne von existentieller Bedeutung. Die Notwendigkeit zur Vorsicht und Verschwiegenheit prägte sowohl die Kommunikationsformen als auch die Struktur des Netzwerkes. Für das Gelingen eines Umsturzes waren die Beteiligten auf viele eingeweihte Unterstützer und Unterstützerinnen angewiesen. Die aus Sicherheitsgründen notwendige Be-

von Eidbruch und Tyrannenmord. In: Es lebe das »Geheime Deutschland «! Claus Schenk Graf von Stauffenberg. Person, Motivation, Rezeption, hrsg. von Jakobus Kaffanke, Edwin Ernst Weber und Thomas Krause, Berlin 2011, S. 107-122, hier S. 109. Vgl. auch Sven Lange, Der Fahneneid. Die Geschichte der Schwurverpflichtung im deutschen Militär, Bremen 2002, S. $112 \mathrm{f}$.

59 Wolfgang Ernst, Der Ruf des Vaterlandes. Das höhere Offizierskorps unter Hitler. Selbstanspruch und Wirklichkeit. Ein Beitrag zur Problematik des Putschversuchs vom 20. Juli 1944, Berlin 1994, S. 202. Das Fernschreiben ist u. a. veröffentlicht in: Jacobsen, Spiegelbild (wie Anm. 2), S. $24 \mathrm{f}$.

60 Generalfeldmarschall Günther von Kluge, Oberbefehlshaber West, verweigerte den Verschwörern, die ihn seit 1942 immer wieder zu gewinnen versucht hatten, am 20. Juli 1944 seine Unterstützung mit den Worten: »wenn das Schwein [...] tot wäre«, zit. nach: Eberhard Zeller, Geist der Freiheit. Der 20. Juli, München 1965, S. 411.

61 Vitzthum, Stauffenberg (wie Anm. 58), S. 108 f. Vgl. auch das 4. Kapitel »Der mißbrauchte Eid - die Fahneneide im III. Reich« in: Lange, Fahneneid (wie Anm. 58), S. 112-146. 
grenzung der Eingeweihten sowie die ebenfalls nötige strikte Reglementierung des Informationsflusses wirkten sich daher außerordentlich hemmend auf die Funktionalität des Netzwerkes aus. Beeinflusst von äußeren hierarchischen Strukturen, verfügte das Netzwerk zwar über eine gewisse Zentralität jedoch nicht über eine eigene strenge Hierarchie. 